\title{
Developing a Depression Inventory for Screening the Fars Ethnicity in
}

\section{Iran}

\author{
Monir Baradaran Eftekhari ${ }^{1}$, Masoumeh Dejman ${ }^{2}$, Ameneh Setareh Forouzan ${ }^{3}$, Katayoun Falahat ${ }^{1}$, \\ Mohsen Shati ${ }^{4}$, Arash Mirabzadeh ${ }^{5}$, Judy Bass ${ }^{6}$ and Zohreh Mahmoodi ${ }^{7, *}$ \\ ${ }^{1}$ Undersecretary for Research and Technology, Ministry of Health and Medical Education, Tehran, Iran \\ ${ }^{2}$ Department of Mental Health, Johns Hopkins Bloomberg School of Public Health, Baltimore, United State \\ ${ }^{3}$ Social Determinants of Health Research Center, University of Social Welfare and Rehabilitation Sciences, Tehran, Iran \\ ${ }^{4}$ Department of Aging, University of Social Welfare and Rehabilitation Sciences, Tehran, Iran \\ ${ }^{5}$ Social Determinants of Health Research Center and Department of Psychiatry, University of Social Welfare and Rehabilitation Sciences, Tehran, Iran \\ ${ }^{6}$ Department of Mental Health, Bloomberg School of Public Health Johns Hopkins University, Baltimore, Maryland, United State \\ ${ }^{7}$ Social Determinants of Health Research Center, Alborz University of Medical Sciences, Karaj, Iran \\ "Corresponding author: PhD, Associate Professor, Social Determinants of Health Research Center, Alborz University of Medical Sciences, Karaj, Iran. Tel: +98-9125474495, Fax: \\ +98-262563341, Email: zohrehmahmoodi2011@gmail.com
}

Received 2018 July 27; Revised 2019 March 17; Accepted 2019 August 20.

\section{Abstract}

Background: Depression is a major public health concern that accounts for a high global disease burden.

Objectives: The aim of this study, developing a depression inventory for screening the Fars ethnicity in Iran.

Methods: The research was carried out using a combined (multi-stage) method in the Persian ethnicity between April 2016 and March 2017. Individual interviews were conducted in Tehran, Iran, with depressed patients, the patients' relatives or friends and key informants. In the next stage, a 28 -item tool was designed. The face, content, criterion and construct validity (confirmatory factor analysis) of the designed tool were assessed in order to determine its validity. Data were analyzed by Amos 24 and spss16.

Results: This study was conducted on 150 individuals, 55\% female and 45\% male, selected from the general public through convenience sampling with a mean age of $35.28 \pm 12$. 38. The impact score for all the items was between 1.8 and 5 , CVR was between 0.7 and 1 for all the items and S-CVI/Ave was $97.09 \pm 0.63$. Based on the results of the confirmatory factor analysis, all the components were approved as factors used to screen for depression. The model fit coefficients, the CMIN/DF was calculated as 1.93. NFI, CFI and GFI were $0.70,0.54$ and 0.73 respectively. For reliability, Cronbach's alpha was calculated as 0.88 with an internal consistency coefficient that is 0.7 .

Conclusions: This depression screening tool for the Fars ethnicity was designed based on the Fars ethnicity and the Iranian culture and appears to be an appropriate tool due to its good reliability, validity, simplicity and applicability in different situations.

Keywords: Depression, Ethnicity, Inventory, Iran

\section{Background}

Depression is a major public health concern that accounts for a high global disease burden; despite its high prevalence and partly due to the high costs of treatment, 90\% of those affected do not receive the needed treatment $(1,2)$. The result of the Iranian Mental Health Survey that was conducted from 2010 to 2011 nationwide, showed that $12.7 \%$ of the general population are depressed (3), However, unlike many other countries in the region, Iran saw a decrease in the percentage of injury-related DALYs (Disabilityadjusted life year) between 1990 and 2010 from $19 \%$ in 1990 to $15 \%$ in 2010 (4) Studies have increasingly shown that ethnic and cultural factors have a major impact on the presentation, diagnosis and assessment of the prevalence of ma- jor depression (5-7).

Presentations of major depression differ by setting and culture and are determined particularly by how bold somatic, emotional or psychological expressions are in that culture (8). In non-western countries, a diagnosis of depression might be morally unacceptable and its clinical manifestation may cause family, religious or social conflicts, as patients and their relatives tend not to consider it a biologically-determined disease and therefore do not seek professional help for it (9) and the recognition of depression becomes complicated.

The lack of culturally-appropriate instruments and the inadequate word-for-word translation of the available screening instruments are among the barriers of screen- 
ing major depression in different cultures, especially in non-western countries. The mere reproduction of questionnaires from one language to another causes misleading and inaccurate interpretations $(10,11)$. Developing and improving screening tools for major depression in different settings, especially in non-western countries, is therefore critical. There is some instrument for screening or diagnostic of depression such as, the geriatric depression scale (GDS), Center for Epidemiological Studies Depression Scale, general health questionnaire, patient health questionnaire (PHQ), the Hamilton depression rating scale (HAM-D), Beck depression inventory, but they each have some limitation; for example GDS is designed to minimize the impact of somatic symptoms associated with aging and illness. Beck inventory is designed to assess the intensity of symptoms associated with psychoanalytic aspects of depression but for certain demographic groups and cultures, they have limited predictive power and validity because of the effects of translation from English to other languages, patients' different interpretations of its emotional terms, and various cultural factors, such as perceptions of racial prejudice and a cultural group's work ethic. These biases are observed with other screening tools used in primary care, including the Center for Epidemiological Studies Depression Scale, designed for diverse demographic groups, and the general health questionnaire and PHQ, which are used to identify short-term changes in mental health (12).

In Iran, too, depression is a dire health issue that needs to be systematically and continuously studied. In a survey of the twelve-month prevalence and severity of psychiatric disorders on people aged 15 - 64 years residing in Iran, 23.6\% met the criteria for at least one DSM-IV psychiatric disorder. The group of anxiety disorders (15.6\%) was the most prevalent group of disorders, followed by mood disorders (14.6\%). The most prevalent DSM-IV disorder was major depressive disorder (12.7\%), followed by generalized anxiety disorder (5.2\%) and obsessive-compulsive disorder (5.1\%); (13).

As a middle eastern country, Iran has used translations of western instruments to screen for depressive disorders. Although some of these translated and adapted questionnaires have demonstrated a suitable validity in various ethnic contexts, considering the differences in cultural values between Western cultures and others, it is still unclear whether a realistic picture has been drawn of this phenomenon in each setting (14) such as Iran.

When designing a tool or questionnaire, the items can be developed through a review of literature on the intended concept, clinical observations and interviews, qualitative research such as grounded theory studies, selections from items in other existing tools or a combination of all these methods (15).

\section{Objectives}

A local inventory was developed of the common symptoms of depression using a standardized translation of Beck's depression inventory. This functional assessment tool was validated with the aim of improving the screening and evaluation of major depression in adults in three major ethnic groups in Iran. This paper presents the validity assessment of the inventory in the Fars ethnicity.

\section{Materials and Methods}

\subsection{Design}

The present study is part of a project aimed to design a tool for screening depression tailored to the Iranian culture in three ethnic groups, namely the Fars, Turk and Azerbaijani ethnicities. This report presents the results of the depression screening tool in the Persian ethnicity. The research was carried out using a combined (multi-stage) method in a qualitative and a quantitative stage.

\subsection{First Stage: Tool Development}

First, in order to investigate the concept and symptoms of depression, individual interviews were conducted in Tehran, Iran, with depressed patients, the patients' relatives or friends and key informants (psychologists/psychiatrists). The inclusion criteria consisted of a self-declared Fars ethnicity, being born in Tehran and speaking Persian. The interviews continued until data saturation occurred and when no new categories of data could be extracted (16). A total of 20 male and female patients with depression entered the study and the samples were from all the different age groups in the 18 - 60 age range. After the content analysis, a list of the signs and symptoms of depression in the patients and their relatives/friends was prepared for the Fars ethnicity. The cases were then finalized after individual interviews with the key informants. In the next stage, a 28 -item tool was designed based on the Likert scale in group discussions held among the research team. This tool examines additional domains over the 21-item Beck depression inventory (BDI) standardized in Iran (17), including beliefs and the use of the social networks or communication devices (cellphone, TV, etc.) (18).

\subsection{Second Stage: Psychometric Assessment of the Tool}

The face, content, criterion and construct validity (confirmatory factor analysis) of the designed tool were assessed in order to determine its validity. Since the purpose of the designed tool was to screen for depression in the society, sampling was carried out in the community. 


\subsubsection{Face Validity}

Face validity was determined using both a qualitative and a quantitative method. To determine the qualitative face validity, ten members of the general public were selected using convenience sampling and face to face interviews were held to examine the level of difficulty of the items (difficulty understanding the statements and words). After modifying the items according to the points of view expressed by the public, a quantitative method (the impact score) was used to reduce the number of the items, eliminate inappropriate ones and determine the importance of each. In the impact score method, if the score was equal to or greater than 1.5 , the item was deemed appropriate for the subsequent analysis and was kept in the tool.

\subsubsection{Content Validity}

The content validity was determined using a qualitative and a quantitative method. First, based on the judgments of knowledgeable experts in tool design and psychologists and psychiatrists, the content validity of the tool was assessed. In the qualitative content validity assessment, the researchers asked ten experts to express their opinions after carrying out a qualitative assessment of the tool in terms of grammar, wording, necessity, importance, item allocation and proper scoring (19). The quantitative content validity was determined using the content validity ratio (CVR) and the content validity index (CVI).To measure the CVR, the said ten experts were asked to rate each item based on a 3-point scale (from 'necessary' to 'not necessary'). Then, if the number obtained from Lawshe's table for determining the minimum value of the index was greater than 0.62 , the item was deemed necessary and important for the tool at the statistical significance level of $P$ $<0.05$ (20). The CVI was evaluated based on the Waltz and Bausell CVI (19). The experts were asked to determine the relevance, simplicity and clarity of each item in the questionnaire. These three criteria were separately assessed by the experts for each item using a 4-point Likert scale. If the CVI of an item was greater than 0.79, the item was deemed appropriate, if the score was 0.75 - 0.80 , the item was deemed questionable, and if the score was less than 0.70 , the item was deemed unacceptable and had to be eliminated from the tool (21). In the next step, based on the mean CVI of all the questionnaire items, the mean scalelevel content validity index/average(S-CVI/Ave) of the questionnaire was calculated. Polit et al. have recommended a score of $90 \%$ or higher for accepting the S-CVI/Ave (22).

\subsubsection{Criterion and Construct Validity}

To determine its criterion validity, this tool was compared with BDI. The correlation coefficient between the scores obtained from the two inventories was calculated as the predictive validity index of the said tool. In this study, the main concept used for designing the depression screening tool was Beck's inventory. Given that this concept was the basis of the development of this tool, a confirmatory factor analysis was used to determine the construct validity.

Determining the minimum sample size required to collect data on structural equation modeling is very important (23). Shah and Goldstein (24) and Schoenberg (25) have recommended a sample size of about 150 for confirmatory factor analysis. In this study, the data of 150 individuals from the general public was collected. Data were analyzed by Amos 24 and SPSS 16 .

\subsection{Third Stage: Reliability of the Tool}

The reliability of the questionnaire was determined using the internal consistency method. Cronbach's alpha is the most commonly-used method for examining the internal consistency of a tool (22). The sample size needed for determining the tool's reliability was estimated as 50 .

\subsection{Ethical Code}

The research project was approved with two codes of ethics -IR.USWR.REC.1395.104 from the University of Social Welfare and Rehabilitation Sciences, and Abzums.Rec.1394.48 from Alborz University of Medical Sciences.

\subsection{Moral Considerations}

The study objectives and obtained their written consent if they were willing to participate in the project data gathering was.

\section{Results}

This study was conducted on 150 individuals selected from the general public through convenience sampling with a mean age of $35.28 \pm 12.38$ (Table 1).

\subsection{Face Validity}

The findings of the qualitative validity assessment led to changes in the content of some of the items, and the impact score for all the items was between 1.8 and 5, and all the items were thus kept for performing the next stage.

\subsection{Content Validity}

In the qualitative content validity assessment, changes were made to the content of some of the items based on the experts' opinions. In the quantitative content validity assessment, CVR was between 0.7 and 1 for all the items, and no item was thus eliminated from the next step. According to the CVI, the mean relevance, mean clarity, mean 
Table 1. The Frequency Distribution of Age, Education, Gender, Marital Status, Income and Number of Family Members in the Participants

\begin{tabular}{|c|c|}
\hline Variable & $\mathbf{F}(\%)$ \\
\hline \multicolumn{2}{|l|}{ Gender } \\
\hline Male & $67(45)$ \\
\hline Female & $82(55)$ \\
\hline \multicolumn{2}{|l|}{ Age } \\
\hline$<20$ & $11(7.4)$ \\
\hline $21-40$ & $96(64.4)$ \\
\hline$\geq 41$ & $42(28.2)$ \\
\hline \multicolumn{2}{|l|}{ Education } \\
\hline Under diploma & $41(27.5)$ \\
\hline BS & $70(47)$ \\
\hline MS & $30(20.1)$ \\
\hline $\mathrm{PhD}$ & $8(5.4)$ \\
\hline \multicolumn{2}{|l|}{ Income } \\
\hline Unpleasant & $28(18.8)$ \\
\hline Almost good & $78(52.3)$ \\
\hline Favorable & $43(28.9)$ \\
\hline \multicolumn{2}{|l|}{ Marriage status } \\
\hline Permanent marriage & $101(67.8)$ \\
\hline Widow & $5(3.4)$ \\
\hline Divorce & $1(0.7)$ \\
\hline Single & $42(28.2)$ \\
\hline \multicolumn{2}{|l|}{ Family member } \\
\hline One person & $6(4)$ \\
\hline Two person & $37(24.8)$ \\
\hline 3- 4 person & $86(57.7)$ \\
\hline$>4$ person & $20(13.4)$ \\
\hline
\end{tabular}

simplicity and S-CVI/Ave were $98.2 \pm 1.9,96.73 \pm 0.70,97.64$ \pm 0.61 and $97.09 \pm 0.63$, respectively. As a result, none of the items were removed and the questionnaire entered the next stage with 28 four-choice items.

\subsection{Construct Validity}

As previously stated, single-factor confirmatory factor analysis was performed in order to confirm the homogeneity of the items in the questionnaire in terms of content and underlying dimensions. There are several goodness-offit indices for evaluating confirmatory factor analysis models. According to the model fit coefficients, the CMIN/DF was calculated as 1.93. In single-factor models, a CMIN/DF less than 3 indicates the favorability of the model (26). The closer are the NFI, CFI and GFI $(0.70,0.54$ and 0.73 respectively) to 1 and the closer is RMSEA (0.79) to zero, the more favorable is the model, and the results obtained in this study show the relatively favorable fit of the model (Table 2).

Based on the results of the confirmatory factor analysis, all the components were approved as factors used to screen for depression (Figure 1). Of these components, item 11 on decision-making power $(0.661)$, item 1 on sadness (0.629) and item 13 on feeling guilty (0.626) had the highest regression weight or impact in terms of depression screening, and item 27 on the use of social networks had the lowest impact (0.292) (Table 3).

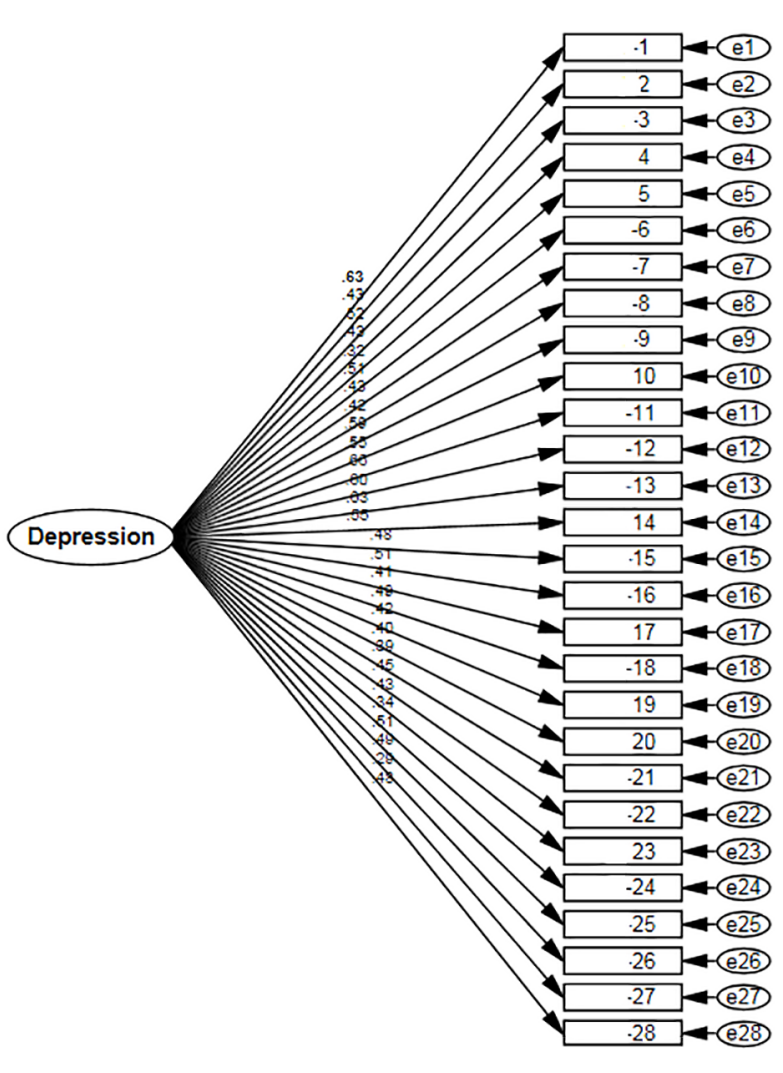

Figure 1. The diagram of the path standardized coefficients of the single-factor structure of FARS depression inventory for screening 1- 28 items

\subsection{Reliability}

Cronbach's alpha was calculated as 0.88. An internal consistency coefficient that is 0.7 or higher is considered acceptable; therefore, the questionnaire was approved for large-scale research (27) (Table 4).

\section{Discussion}

The study of any subject requires a suitable tool for collecting the required information with utmost precision 


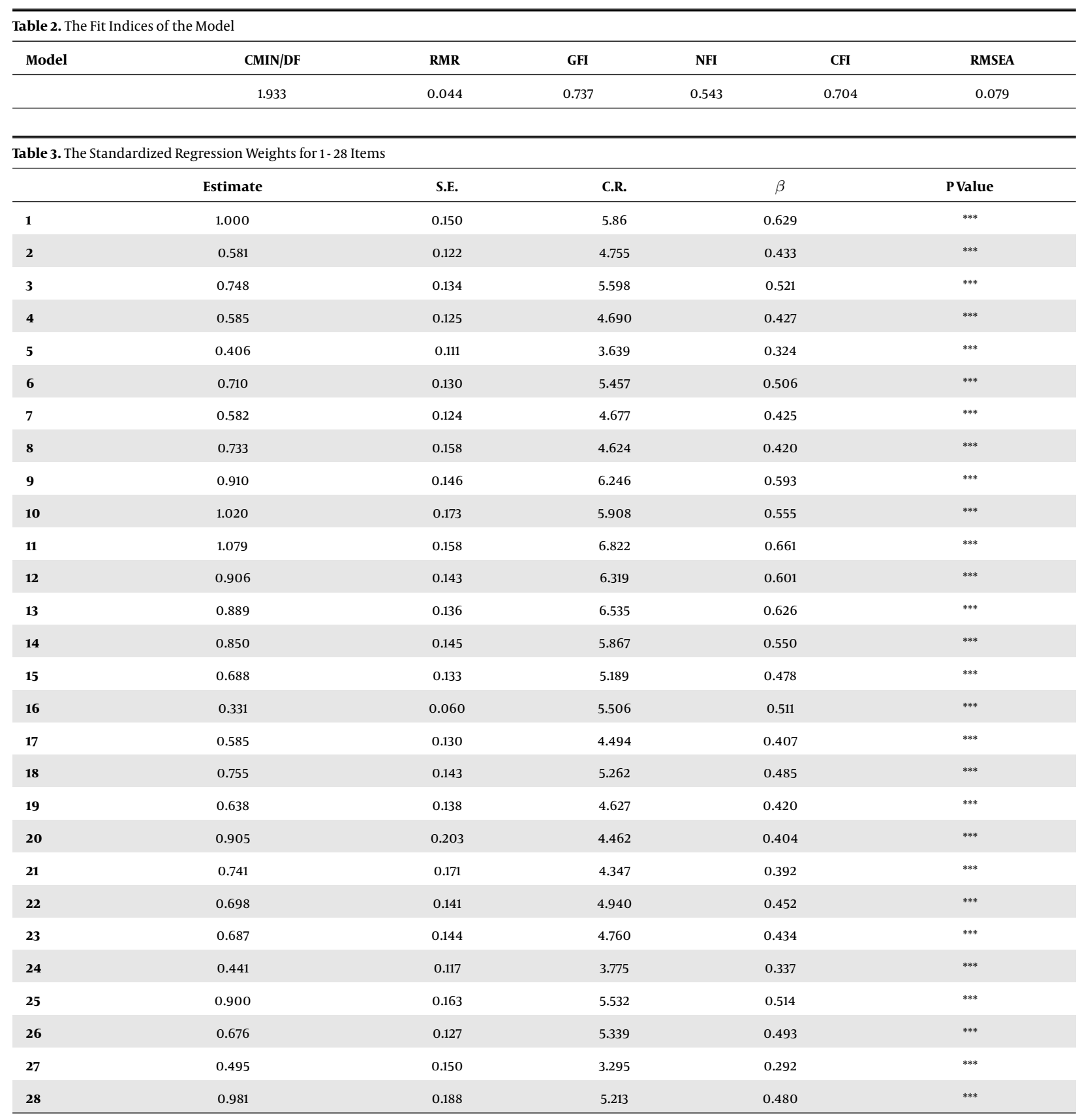

\begin{tabular}{lc}
\hline Table 4. Reliability Statistics & \\
\hline Cronbach's Alpha & No. of Items \\
\hline 0.888 & 28 \\
\hline
\end{tabular}

and the least amount of error (28). A tool designed in a particular country only reflects the culture of that country, and if used elsewhere, even if by way of a precise translation, it may be problematic due to the content not being a good fit (29).

The present study was conducted to assess the psychometric properties of a depression screening tool for the Fars ethnicity in Iran. The results of the study confirmed the validity and reliability of the tool. The face and content validity, simplicity and clarity of the items were also confirmed. The evidence on the "relevance" of the items in the CVI showed a significant degree of agreement between the experts $($ mean $=98.2 \%$ ). 
The results of the single-factor confirmatory factor analysis showed the good fit of the model. In this tool, sadness and feeling guilty were the variables with the greatest impact on depression screening. The review of literature showed no studies on the effects of each of the signs of depression on its incidence. There were also no studies that had investigated the cross-cultural and crosslanguage characteristics of depressed speech (30).

Nonetheless, recent studies suggest that, in addition to studying the mean scores, the emphasis in research has to be on the dynamic relationships between the signs and symptoms of depression (31). Sepehrmanesh and Moraveji found that despair and suicidal thoughts are the most common depression symptoms (32). Bringmann et al. reported the absence of pleasure and discontent as the most central components of the BDI (31). Ethnic differences likely affect the formation and expression of depression symptoms $(33,34)$. Therefore, knowing which item has the biggest impact on the score obtained in a screening tool can contribute to the effective use of the tool for a better and faster diagnosis by service providers.

In this study, Cronbach's alpha coefficient of the designed questionnaire showed a high internal consistency among the items, which confirms the reliability of the depression screening tool for the Fars ethnicity in Iran.

In depression, the way the affected person presents his symptoms can be fundamentally different from the symptoms with which the disease is manifested or presented. The symptoms expressed by the patient depend on his perception of the disease and judgment and responses to psychological symptoms, while the symptoms manifested by the patient are concerned with the psychiatrist's judgement and the patient's condition during the clinical examination and interview (35). Unlike the BDI, the tool designed based on the findings of this qualitative study in the Fars ethnicity of Iran has 28 items that have been designed based on the descriptions of depression symptoms within the language and cultural context of Iran. In addition, this tool contains seven added items that are not measured in the BDI: Too much thinking, Beliefs, thinking of death, being sensitive, self-confidence, use of the social networks and communication devices. For instance, the use of social networks is one of the items that were mentioned in the interviews. Although it had the lowest impact in terms of screening for depression, the patients discussed it as a symptom of depression due to the changes that have recently taken place in societies. A strength of this tool was its use of the opinions of the patients and their relatives/friends, who were directly involved with the presenting symptoms (Supplementary File Appendix 1)

\subsection{Limitations}

The lack of studies on the effect of each depression symptom on depression screening limited the further comparison of the results with other studies.

\subsection{Conclusions}

This depression screening tool for the Fars ethnicity was designed based on the Fars ethnicity and the Iranian culture and appears to be an appropriate tool due to its good reliability, validity, simplicity and applicability in different situations. The researchers hope that this tool can be used by general practitioners or psychologists in health screening centers to help improve the timely diagnosis of depression, which is a prevalent but underdiagnosed disease in these centers. The designed tool is recommended to be used to evaluate the impact of interventions on depression treatment in psychologic intervention studies or in minimal surveys in Persian-speaking groups.

\section{Supplementary Material}

Supplementary material(s) is available here [To read supplementary materials, please refer to the journal website and open PDF/HTML].

\section{Footnotes}

Authors' Contribution: Monir Baradaran Eftekhari, Masoumeh Dejman and Ameneh Setareh Forouzan Studied and designed the concept. Katayoun Falahat collected the data. Mohsen Shati analysed and interpretated the data. Arash Mirabzadeh, Judy Bass and Zohreh Mahmoodi drafted the manuscript. Zohreh Mahmoodi revised the manuscript Critically for important intellectual content. Mohsen Shati analysed the data statistically.

Declaration of Interests: None to declare.

Ethical Approval: The research project was approved with two codes of ethics -IR.USWR.REC.1395.104 from the University of Social Welfare and Rehabilitation Sciences, and Abzums.Rec.1394.48 from Alborz University of Medical Sciences.

Funding/Support: University of Social Welfare and Rehabilitation Sciences, and Alborz University of Medical Sciences.

\section{References}

1. Kohn R, Saxena S, Levav I, Saraceno B. The treatment gap in mental health care. Bull World Health Organ. 2004;82(11):858-66. [PubMed: 15640922]. [PubMed Central: PMC2623050]. 
2. Wang PS, Angermeyer M, Borges G, Bruffaerts R, Tat Chiu W, D. E. Girolamo G, et al. Delay and failure in treatment seeking after first onset of mental disorders in the World Health Organization's World Mental Health Survey Initiative. World Psychiatry. 2007;6(3):177-85. [PubMed: 18188443]. [PubMed Central: PMC2174579].

3. Rahmanian H, Mohammadinasab A, Duncko R. Depression in Iran globally minded: Volunteering and international psychiatry special interest group. 2017. Available from: https://www.globallyminded.org/ home/depression-in-iran/.

4. Shahraz S, Forouzanfar MH, Sepanlou SG, Dicker D, Naghavi P, Pourmalek F, et al. Population health and burden of disease profile of Iran among 20 countries in the region: From Afghanistan to Qatar and Lebanon. Arch Iran Med. 2014;17(5):336-42. [PubMed: 24784862].

5. Alegria M, Chatterji P, Wells K, Cao Z, Chen CN, Takeuchi D, et al. Disparity in depression treatment among racial and ethnic minority populations in the United States. Psychiatr Serv. 2008;59(11):1264-72. doi: 10.1176/appi.ps.59.11.1264. [PubMed: 18971402]. [PubMed Central: PMC2668139].

6. Bernert S, Matschinger H, Alonso J, Haro JM, Brugha TS, Angermeyer MC, et al. Is it always the same? Variability of depressive symptoms across six European countries. Psychiatry Res. 2009;168(2):13744. doi: 10.1016/j.psychres.2008.04.008. [PubMed: 19481817]. [PubMed Central: PMC3635143]

7. Schrier AC, de Wit MA, Rijmen F, Tuinebreijer WC, Verhoeff AP, Kupka RW, et al. Similarity in depressive symptom profile in a populationbased study of migrants in the Netherlands. Soc Psychiatry Psychiatr Epidemiol. 2010;45(10):941-51. doi: 10.1007/s00127-009-0135-0. [PubMed: 19763363].

8. Kirmayer LJ. Psychotherapy and the cultural concept of the person. Transcult Psychiatry. 2007;44(2):232-57. doi:10.1177/1363461506070794. [PubMed: 17576727].

9. Deisenhammer EA, Coban-Basaran M, Mantar A, Prunnlechner R, Kemmler G, Alkin T, et al. Ethnic and migrational impact on the clinical manifestation of depression. Soc Psychiatry Psychiatr Epidemiol. 2012;47(7):1121-9. doi: 10.1007/s00127-011-0417-1. [PubMed: 21805303].

10. Jordans MJ, Komproe IH, Ventevogel P, Tol WA, de Jong JT. Development and validation of the child psychosocial distress screener in $\mathrm{Bu}-$ rundi. Am J Orthopsychiatry. 2008;78(3):290-9. doi: 10.1037/a0014216. [PubMed: 19123748]

11. Kohrt BA, Tol WA, Harper I. Reconsidering somatic presentation of generalized anxiety disorder in Nepal. $J$ Nerv Ment Dis. 2007;195(6):544. author reply 545. doi: 10.1097/NMD.ob013e318064e7eb. [PubMed: 17568305].

12. Kerr LK, Kerr LD Jr. Screening tools for depression in primary care: the effects of culture, gender, and somatic symptoms on the detection of depression. West JMed.2001;175(5):349-52. doi:10.1136/ewjm.175.5.349. [PubMed: 11694495]. [PubMed Central: PMC1071624].

13. Sharifi V, Amin-Esmaeili M, Hajebi A, Motevalian A, Radgoodarzi R, Hefazi M, et al. Twelve-month prevalence and correlates of psychiatric disorders in Iran: The Iranian Mental Health Survey, 2011. Arch Iran Med. 2015;18(2):76-84. [PubMed: 25644794].

14. Kaiser BN, Kohrt BA, Keys HM, Khoury NM, Brewster AR. Strategies for assessing mental health in Haiti: Local instrument development and transcultural translation. Transcult Psychiatry. 2013;50(4):532-58. doi 10.1177/1363461513502697. [PubMed: 24067540].

15. Frank-Stromborg M. Instruments for clinical health-care research. Jones \& Bartlett Learning; 2004

16. Marshall B, Cardon P, Poddar A, Fontenot R. Does sample size matter in qualitative research?: A review of qualitative interviews in is research. J Comput Inf Syst. 2015;54(1):11-22. doi: 10.1080/08874417.2013.11645667.

17. Rajabi G, Karjo KS. Psychometric properties of a persian-language version of the Beck Depression Inventory-second edition(BDI-II-Persian). Train Meas. 2013;3(10):139-57.

18. Dejman M, Mahmoodi Z, Baradaran Eftekhari M, Forouzan AS, Fala- hat $\mathrm{K}$, Shati M, et al. Explaining experiences of depression in cities representing the Fars, Turk and Kurd ethnic groups of Iran: A qualitative study. Iran J Psychiatry Behav Sci. 2018;12(4). e66984. doi: 10.5812/ijpbs.66984.

19. Mehmannavazan M, Hosseini M, Vartanoosian J, Matbouei M, Nasiri M, Vasli P. Translation, cultural adaptation and preliminary psychometric evaluation of the "Family Management Measure" among Iranian families with a child with a chronic disease. Electron Physician. 2018;10(6):6942-50. doi: 10.19082/6942. [PubMed: 30034662]. [PubMed Central: PMC6049973].

20. Lawshe $\mathrm{CH}$. A quantitative approach to content validity. Pers Psychol. 1975;28(4):563-75. doi: 10.1111/j.1744-6570.1975.tb01393.x.

21. Polit DF, Beck CT, Owen SV. Is the CVI an acceptable indicator of content validity? Appraisal and recommendations. Res Nurs Health. 2007;30(4):459-67. doi: 10.1002/nur.20199. [PubMed: 17654487].

22. Polit D, Beck C, Hungler B. Essentials of nursing research: Methods, appraisal, and utilization. Philadelphia, PA: Lippincot Williams \& Wilkins; 2006.

23. McQuitty S. Statistical power and structural equation models in business research. J Bus Res. 2004;57(2):175-83. doi: 10.1016/s01482963(01)00301-0.

24. Shah R, Goldstein SM. Use of structural equation modeling in operations management research: Looking back and forward. J Oper Manag. 2006;24(2):148-69. doi: 10.1016/j.jom.2005.05.001.

25. Schoenberg R. Covariance structure models. Annu Rev Sociol. 1989;15(1):425-40. doi: 10.1146/annurev.so.15.080189.002233.

26. Maat SM, Adnan M, Abdullah MFNL, Ahmad CNC, Puteh M. Confirmatory factor analysis of learning environment instrument among high performance school students. Creat Educ. 2015;6(6):640-6. doi: 10.4236/ce.2015.66063.

27. Gliem JA, Gliem RR. Calculating, interpreting, and reporting Cronbach's alpha reliability coefficient for Likert-type scales. Midwest Research-to-Practice Conference in Adult, Continuing, and Community. 2003.

28. Eslami M, Heidania A, Heidarzade A, Shakouri Farkhonde A, Motlagh ME. [Designing and determining validity and reliability of the questionnaire for the effect of HBM on users of two methods of birth control with pills and condoms]. Oromieh J. 2011;21(5):382-90. Persian.

29. Doward LC, Meads DM, Thorsen H. Requirements for quality of life instruments in clinical research. Value Health. 2004;7 Suppl 1:S13-6. doi: 10.1111/j.1524-4733.2004.7s104.x. [PubMed: 15367238].

30. Alghowinem S, Goecke R, Epps J, Wagner M, Cohn J. Cross-cultural depression recognition from vocal biomarkers. Interspeech. 2016. p. 1943-7.

31. Bringmann LF, Lemmens LH, Huibers MJ, Borsboom D, Tuerlinckx F. Revealing the dynamic network structure of the Beck Depression Inventory-II. Psychol Med. 2015;45(4):747-57. doi: 10.1017/S0033291714001809. [PubMed: 25191855].

32. Sepehrmanesh Z, Moraveji A. Prevalence of depression among interns of Kashan University of Medical Science in 2013. Eur Psychiatry. 2015;30:718. doi: 10.1016/s0924-9338(15)30568-x.

33. Assari S. Ethnic and gender differences in additive effects of socioeconomics, psychiatric disorders, and subjective religiosity on suicidal ideation among blacks. Int J Prev Med. 2015;6:53. doi: 10.4103/20087802.158913. [PubMed: 26180624]. [PubMed Central: PMC4498310].

34. Assari S, Dejman M, Neighbors HW. Ethnic differences in separate and additive effects of anxiety and depression on self-rated mental health among blacks. J Racial Ethn Health Disparities. 2016;3(3):423-30. doi: 10.1007/s40615-015-0154-3. [PubMed: 27294736].

35. Kessler RC, Berglund P, Demler O, Jin R, Koretz D, Merikangas KR, et al. The epidemiology of major depressive disorder: Results from the National Comorbidity Survey Replication (NCS-R). JAMA. 2003;289(23):3095-105. doi: 10.1001/jama.289.23.3095. [PubMed: 12813115]. 